317 | juillet-septembre 1999

Numéro spécial : France-Allemagne. Interactions,

références

\title{
La Révolution française et l'Allemagne : du paradigme comparatiste à la recherche des transferts culturels
}

\section{Matthias Middell}

\section{(2) OpenEdition}

\section{Journals}

Édition électronique

URL : https://journals.openedition.org/ahrf/266

DOI : 10.4000/ahrf.266

ISSN : 1952-403X

Éditeur :

Armand Colin, Société des études robespierristes

Édition imprimée

Date de publication : 1 septembre 1999

Pagination : 427-454

ISSN : 0003-4436

Référence électronique

Matthias Middell, «La Révolution française et l'Allemagne: du paradigme comparatiste à la recherche des transferts culturels », Annales historiques de la Révolution française [En ligne], 317 | juillet-septembre 1999, mis en ligne le 10 avril 2006, consulté le 22 avril 2022. URL : http://journals.openedition.org/ ahrf/266 ; DOI : https://doi.org/10.4000/ahrf.266

Ce document a été généré automatiquement le 22 avril 2022.

Tous droits réservés 


\title{
La Révolution française et l'Allemagne : du paradigme comparatiste à la recherche des transferts culturels
}

\author{
Matthias Middell
}

1 L'objectif de cette présentation n'est pas de proposer une bibliographie récente et complète des livres et articles traitant de la Révolution de 1789 et des réponses allemandes au "printemps des peuples». Il nous importe beaucoup plus de préciser une nouvelle direction d'interprétation d'importance grandissante ${ }^{1}$. Il s'agit plus précisément de la modification de la perspective qui faisait de la Révolution française le point de départ (et parfois la norme) de la comparaison entre les évolutions nationales à la fin du XVIIIe et au XIX ${ }^{e}$ siècle. Cette perspective déjà ancienne repose sur une question qui avait occupé les contemporains de la Révolution française et qui avait pris dans l'historiographie libérale du début $\mathrm{du} \mathrm{xIX}^{\mathrm{e}}$ siècle, tout comme dans la théorie sociale marxiste, une place centrale. Formulons la ainsi : de quelle manière peuvent être réglés au mieux les problèmes de la transition entre une société d'Ancien Régime organisée en « états " principalement agricoles et tenant sa cohésion de l'absolutisme d'une part et une société reposant sur les principes de la souveraineté populaire, de l'individualisme, de la liberté et (en partie) de l'égalité d'autre part ? À ce point de vue s'ajoute l'évaluation des conséquences de cette transition pour la formation de la société bourgeoise qui allait suivre.

2 On sait que cette perspective est liée aux luttes politiques du XIX ${ }^{e}$ siècle, et à celles aussi d'une bonne part du $\mathrm{xx}^{\mathrm{e}}$ siècle. C'est pourquoi la Révolution française a sans cesse été un sujet d'affrontements ardents. La concentration des moyens de recherche et de matière grise a ainsi permis des innovations méthodologiques qui ont finalement eu des répercussions sur d'autres domaines de l'historiographie, que ce soit en histoire politique, en histoire sociale ou des mentalités, que ce soit sur le terrain de l'étude de la culture politique ou enfin sur celui des symboles. Ces deux aspects, les avancées 
méthodologiques et l'intérêt politique, ont conféré à l'historiographie de la Révolution française une dimension internationale supérieure à d'autres sujets d'écriture de l'histoire.

3 Si nous prenons en compte le caractère international des réactions suscitées par la prise de la Bastille et l'exécution du roi [d'Edmund Burke à Georg Forster ${ }^{2}$ ], si nous considérons le débat européen du Vormärz et sur les comparaisons à l'intérieur du triangle européen franco-germano-britannique ${ }^{3}$, nous pouvons dire qu'à partir de la fin du XIX ${ }^{e}$ siècle et plus particulièrement depuis la Seconde Guerre mondiale le débat sur la Révolution française est passé d'un statut franco-français à un statut international. Certes la dominante reste française, mais nous notons des points forts en Angleterre, aux États-Unis, en Italie, en Espagne, en Russie, et, pour une moindre part, également dans les Balkans, en Asie, en Afrique, en Amérique latine et dans divers pays socialistes de l'Europe de l'Est ${ }^{4}$.

4 Partout dans le monde, la Révolution française a joué un rôle central dans l'évaluation contrastive des voies menant à l'époque actuelle. L'Allemagne pour sa part a joué sous certains aspects un rôle plus particulier. Dès le xvIII siècle, beaucoup d'intellectuels y présentent leur pays comme le contrepoint des évolutions françaises. Cette position se fait jour dans l'idée d'un certain retard de l'Allemagne dans le processus menant à la révolution de 1848 ou encore dans l'idée d'un caractère national allemand réformateur par contraste avec le caractère français révolutionnaire à l'époque de «l'ennemi héréditaire ».

5 Après la Seconde Guerre mondiale, la mémoire collective allemande se concentre sur l'analyse des immenses crimes du national-socialisme et de l'holocauste. Dans les deux États allemands, les chercheurs ont recours à l'idée de "spécificité allemande", qui reprend la vieille tradition tant du libéralisme allemand que du marxisme d'un retard en comparaison de la France issue de la tradition révolutionnaire, en conformité avec les interprétations des alliés. Ce modèle de la "spécificité allemande » associe l'absence d'une révolution bourgeoise démocratique, la faiblesse structurelle de la bourgeoisie libérale et la persistance des structures traditionnelles de la vieille noblesse, de la caste militaire et du respect de l'autorité établie, respect forgé dans l'absolutisme. Ce modèle s'est imposé comme schéma central d'explication dans les études historiques en Allemagne. Ainsi, depuis les années soixante, les partisans d'une histoire sociale critique ont pris le dessus en R.F.A. en mettant leurs considérations sur l'histoire de la société en rapport direct avec la thèse de la "spécificité allemande ». Toutefois il faut noter que ces historiens ont souligné la nécessité de sortir d'une spécificité désormais problématique avec le caractère effectif de la démocratisation ${ }^{5}$. De même, en R.D.A., une historiographie marxiste-léniniste nouvelle s'est efforcée de montrer qu'il existe des solutions de remplacement aux évolutions malheureuses en Allemagne (et parmi ces solutions en premier lieu aussi les «jacobins allemands » des années 1790) et a essayé en conséquence de développer pour son propre pays un autre schéma, permettant de sortir in fine de la "spécificité allemande». Les conséquences contradictoires de ces démarches ont nourri bien des controverses entre les historiens des deux États. Pourtant les schémas généraux d'interprétation historique étaient bien plus proches les uns des autres que les adversaires idéologiques voulaient le concéder ${ }^{6}$. La Révolution française a une place centrale dans ces discours, comme solution manquée au départ de parcours divergents. Pourtant, ou peut-être pour cette raison, l'étude véritable de l'histoire de la Révolution resta du domaine de quelques experts, 
qui, de plus, n'avaient souvent dans les débats historiographiques qu'une audience faible par rapport aux spécialistes de l'histoire nationale. La Révolution française jouait un rôle dans le schéma comparatiste lié à un imaginaire collectif de l'identité historique, si bien qu'une trop grande connaissance du détail de l'histoire pouvait devenir gênante, même s'il demeure nécessaire qu'un tout petit groupe d'historiens s'occupe malgré tout de l'objet de comparaison en lui-même.

Dans les années quatre-vingt, on vit apparaître les premiers changements. D'abord s'amorce un changement de génération, qui s'amplifie dans la décennie suivante : pour ces jeunes historiens, la confrontation avec le nazisme n'était plus l'objet central des études historiques. Pour eux, une recherche vraiment comparatiste ${ }^{7}$, avec des résultats permettant des différenciations, devient une expérience courante ${ }^{8}$. Ils ne s'avérèrent pas vraiment satisfaits, en particulier dans le cadre du bicentenaire, d'une approche simpliste de la Révolution française comme alternative du développement historique allemand. Il y eut donc une conjonction productive entre les attentes du public en matière d'images de l'histoire et l'arrivée sur le plan international d'une nouvelle génération d'historiens professionnels, qui adoptent d'abord un profil prudent, avant de s'imposer. L'Allemagne constitue en quelque sorte le grand terrain de manœuvre pour ces changements, car les transformations politiques, sociales et culturelles qu'entraîna la Révolution de 1989 ont accéléré ou explicité des processus qui, pour être présents dans les pays voisins de l'Europe de l'Est et de l'Ouest, s'y déroulent sans doute plus lentement.

8 Précisons les aspects qui caractérisent la situation en Allemagne, sur la base d'une disparition progressive de la concurrence entre deux historiographies toutes deux allemandes.

D'abord les points forts des études historiques est-allemandes perdent leur légitimation, en particulier la branche comparatiste reposant sur le concept d'histoire mondiale comme histoire des révolutions. Ce concept avait eu toute sa valeur opératoire, surtout dans les années cinquante et soixante. Il ne faut pas oublier que la découverte des traditions révolutionnaires (dans l'étude des Jacobins allemands par Hedwig Voegt et Heinrich Scheel par exemple) ${ }^{9}$ dans l'histoire allemande puise sa source en son sein. S'y associe l'apparition en Allemagne, pour la première fois, d'une histoire sociale de la Révolution française écrite de manière professionnelle (surtout les études bien connues de Walter Markov sur les mouvements des sans-culottes et les Jacquesroutins) ${ }^{10}$.

$10 \mathrm{Au}$ départ tout observateur averti pouvait constater une différence profonde avec la recherche en Allemagne de l'Ouest, puis celle-ci, à partir des années soixante, comble son retard. Dans la période suivante les analyses expérimentent des deux côtés à l'intérieur de cadres théoriques différents : d'une part l'étude de la bourgeoisie afin de saisir le sens profond de la spécificité allemande ${ }^{11}$, d'autre part l'histoire comparative des révolutions ${ }^{12}$. Malgré toutes ces différences, on ne saurait nier des éléments de convergence, en premier lieu des bases épistémologiques semblables, à savoir la mise en regard des voies spécifiques nationales et l'analyse de leurs composantes. Au milieu des années quatre-vingt, ce paradigme était presque totalement épuisé : un tournant culturel s'impose dans les deux Allemagne sous la forme d'une irruption de nouveaux thèmes et la remise en question prudente du rôle secondaire des perceptions subjectives face aux structures « objectives » dans l'économie et le social. 
11 L'écroulement de la R.D.A. et les transformations des études historiques ont masqué pour un temps le conflit méthodologique, qui a resurgi avec d'autant plus d'âpreté au milieu des années quatre-vingt-dix, en liaison avec le changement de génération et la possibilité de coopérations internationales. La célébration du bicentenaire a assurément accéléré le processus, grâce au contact intensif avec les groupes de recherches de pointe à l'étranger sur l'histoire des mentalités, l'histoire de la culture politique ou l'apparition de l'homme moderne, pour ne citer que quelques aspects de grands colloques scientifiques, grâce aussi à la publication accélérée d'études nouvelles, grâce enfin aux ressources mises à la disposition de la recherche par la fondation Volkswagen et la Deutsche Forschungsgemeinschaft (Conseil national pour la Recherche), les instruments les plus importants du financement des projets de recherche.

12 À l'épreuve de ce comparatisme véritable qui a confronté la recherche allemande aux résultats obtenus par les étrangers sur leurs pays ${ }^{13}$, l'ancien type de recherche, autour de la "spécificité allemande» ou d'une voie spécifique allemande, s'est amenuisé tendant à disparaître au profit d'analyses plus fines qui mettent en avant les interactions plus que la construction des différences.

13 En fin de compte nous trouvons une mise en question inévitable des anciens modèles de comparaison et un intérêt croissant pour les phénomènes d'interaction des histoires des différents lieux et des différentes cultures. En quelques années, abandonnant peu à peu la question des différences structurelles et des conséquences entraînées par le choix d'une voie nationale pour le passage à la société bourgeoise, la recherche sur la Révolution française a imposé le problème de la réception de l'autre, de l'influence réciproque de la sémantique historique ${ }^{14}$, des cultures politiques, des identifications, bref, elle s'est placée dans une perspective de transfert culturel et a pratiqué dans ce champ de multiples expériences.

14 Mais cette nouvelle mise en perspective n'est certes pas encore achevée. Il serait vraiment trop simple d'établir un lien direct entre la remise en cause de l'État national et la rhétorique européenne à la mode d'une part, et ces nouvelles tendances d'une historiographie qui met en relief des similitudes souvent cachées par rapport aux différences longtemps mises en exergue d'autre part. Pourtant, même si les liens sont peut-être plus subtils qu'il n'apparaît au premier abord, ils ne peuvent être niés. Le contact permanent entre des représentants de cultures historiographiques différentes dans des travaux communs est une expérience relativement nouvelle, source d'expériences profondes. Les anciens modèles n'ont pas perdu totalement leur pouvoir explicatif dans la mesure où les résultats acquis sont trop importants pour cela et les méthodes avaient été longuement élaborées. Ce sont plutôt les attentes d'explication qui se sont modifiées. Les vieilles réponses ne sont pas devenues fausses, même si parfois la polémique historiographique use d'un tel argument. Simplement elles ne correspondent plus aux questions qui ont pris de l'importance aujourd'hui.

15 Cette nouvelle perspective de recherche doit son développement rapide en grande partie aux transformations qui ont eu lieu dans le paysage historiographique depuis la fin des années quatre-vingt. Il convient donc d'abord de considérer l'apparition de la critique dirigée contre les vieux modèles d'explication, puis d'évoquer comment s'est formulé le nouveau programme de recherche et enfin de montrer quelques exemples d'application.

I - Le concept de transfert culturel comme réaction à des aspects insatisfaisants de la comparaison historique 
Dès 1981, l'historien Rolf Reichardt, de Mayence, remet en question dans une courte postface l'ensemble des contributions d'un colloque franco-allemand centré sur le bilan des thèmes de comparaison et des relations franco-allemandes à l'époque de la Révolution française. Dans son plaidoyer en faveur des résultats de recherches récentes de part et d'autre du Rhin, il s'en prend à l'idée de la Révolution française comme instrument de mesure de la "spécificité allemande ». Il critique en particulier trois aspects: d'abord l'idéalisation par les historiens de leurs représentations de la Révolution française comme réalisation des Lumières; ensuite le fait de confondre la France et Paris; enfin le fait de déduire du succès d'une révolution bourgeoise la puissance de la bourgeoisie ${ }^{15}$. Ce court texte ne présente pas encore les signes du passage de la notion de comparaison à celle de transfert culturel, mais il faut néanmoins le considérer comme une brèche dans le système ${ }^{16}$.

L'opposition traditionnelle d'une France comme lieu des Lumières sur le plan politique, d'un jacobinisme qui s'impose et d'une bourgeoisie prête à faire la révolution d'une part, et d'une Allemagne qui ne présente aucun de ces aspects, est ici remise en cause. Qui plus est, l'argumentation s'appuie sur les résultats de recherches récentes en histoire sociale et non sur leur négation ${ }^{17}$. Il est intéressant de considérer qu'à la même époque, en Allemagne de l'Est, Manfred Kossok suit une voie identique dans ses réflexions sur les rapports entre réforme et révolution, toujours dans le but de sortir des vieilles confrontations ${ }^{18}$.

18 Alors que, dans un premier temps, les résultats de la recherche sur l'histoire sociale des Lumières, de la société rurale française et du jacobinisme ouvrent de nouvelles perspectives, un colloque à Bielefeld en 1985 marque un tournant majeur par l'apport d'une nouvelle interprétation de la Révolution comme rupture dans la conscience sociale : il repose sur une collaboration étroite entre chercheurs français, américains et allemands et un travail interdisciplinaire exemplaire qui concerne la sémantique historique, l'histoire du discours, la compréhension du temps, la sociabilité, la presse, la littérature révolutionnaire et le monde des symboles et des mythes ${ }^{19}$. Une perspective culturelle prend alors la place d'une interprétation de la révolution principalement politique et sociale. Quelques années plus tard, dans un grand compte rendu des publications, Rolf Reichardt montre en quoi cette nouvelle manière de voir les choses s'impose progressivement.

19 Certes avec un peu de retard vis-à-vis des recherches en R.D.A. sur la Révolution française en elle-même se développe donc en Allemagne de l'Ouest une historiographie très active sur le plan international; elle est le fait d'une nouvelle génération d'historiens et se répartit sur plusieurs centres ${ }^{20}$. C'est ce qui permit à la comparaison sur laquelle reposait la construction d'une "spécificité allemande » de s'accompagner d'une étude empirique des objets de comparaison. Les points forts se déplacent: ce n'est plus le "mûrissement" abstrait de groupes sociaux définis et de formations politiques qui doit distinguer les deux voies menant à l'époque moderne, mais la manière dont les acteurs historiques se représentent les nouvelles formes de la politique et des transformations culturelles et sociales.

20 Cette comparaison historico-culturelle a toutefois ses dangers et ses limites. Michel Espagne met ainsi en relief les limites du comparatisme en histoire culturelle dans un article très discuté depuis sa parution par les historiens allemands ${ }^{21}$. En premier lieu il fait valoir que « le comparatisme présuppose des aires culturelles closes pour se donner les possibilités d'en dépasser ultérieurement les spécificités grâce à des catégories 
abstraites ${ }^{22}$ ». Les interférences qui existent entre les aires culturelles et les groupes sociaux ne se limitent donc pas aux ensembles synchroniques qui sont objet d'attention pour la pratique comparatiste : il faut considérer aussi les aspects hors synchronie. À la suite de son interrogation sur l'importance des interférences qui résultent d'échanges ponctuels mais permanents, le germaniste français conclut que le comparatisme construit des identités sans mettre en relief la part étrangère (souvent cachée), qui existe dans la mémoire nationale. Cette critique s'appuie sur une réflexion liée à un projet de recherche démarré en 1985 à Paris, et appliqué à l'histoire franco-allemande au $\mathrm{XIX}^{\mathrm{e}}$ siècle ${ }^{23}$. Le concept de comparatisme a été ainsi au centre d'une série de coopérations franco-allemandes dans ces dix dernières années où il a pu s'affiner progressivement sur la base de travaux empiriques amples, donc aptes à en mesurer la capacité heuristique.

21 Les divers participants à cette coopération ont des points de départ très différents, leurs intérêts ne sont pas congrus, leurs concepts ne sont pas plus identiques que les méthodes de recherche combinées et les traditions historiques revendiquées, les contextes institutionnels et disciplinaires divergent énormément. Mais tous ces concepts différents ont un intérêt commun pour les procès d'échange culturel, leur motivation, leurs formes, leur portée et leurs conséquences à long terme. Le caractère expérimental de ce domaine de recherche qui a pu se soustraire jusqu'ici à la formulation canonique de sa base épistémologique et à sa transformation en une vulgate, le rend particulièrement attirant pour les historiens culturels. Ainsi, en peu d'années, l'examen des transferts culturels s'est établi en plus de la comparaison historique comme un instrument majeur de la science historique.

22 L'idée décisive qui distingue ce concept des plus anciens, est la suivante : «Les besoins spécifiques du système d'accueil opèrent une sélection : ils refoulent des idées, des textes et des objets, qui demeurent désormais dans un espace où ils restent éventuellement disponibles pour de nouvelles conjonctures [...] la référence à l'autre a le plus souvent une fonction de légitimation ou de subversion à l'intérieur de la culture d'accueil [...]. Il n'est pas même nécessaire d'avoir des connaissances réelles d'une culture étrangère pour tenter de s'en servir ${ }^{24}$ ». Cette "suprématie » de la culture d'accueil en comparaison de la culture d'où émanent les signes culturels modifie la perspective de cette histoire culturelle désormais capable de s'intégrer les résultats de champs de recherche aussi divers que l'histoire des migrations ${ }^{25}$, l'histoire du transfert des objets [(livres ${ }^{26}$, marchandises de consommation ${ }^{27}$ et de luxe, objets d'art ${ }^{28}$ etc.] et l'histoire des idées et des médias de sa diffusion ${ }^{29}$.

23 Hans-Jürgen Lüsebrink et Rolf Reichardtont commencé par l'exploration du monde des symboles, des images et du langage de la Révolution, ainsi que de sa perception en Allemagne ce qui s'est avéré un accès important à la nouvelle perspective. Le premier résultat de ces examens est l'analyse de la prise de la Bastille en tant que symbole collectif ${ }^{30}$ et mythe fondateur. Ce symbole historique majeur se montre particulièrement approprié à représenter la Révolution d'une triple manière :

24 - il explique dans sa bipolarité la conscience de la césure et l'idée de la rupture radicale entre l'Ancien Régime et la Révolution et a donc une valeur type dans des contextes différents ;

25 - il associe d'une manière convaincante une histoire concrète à un imaginaire social général ; 
26 - il ne peut être analysé uniquement sous forme de représentations dans la mesure où sa fonction d'appel aux émotions provoque des réactions collectives qui motivent et influencent l'action.

27 Lüsebrink et Reichardt mettent aussi en évidence les différentes dimensions de la diffusion de ce symbole collectif: dans les démonstrations graphiques, comme argument politique, comme élément narratif, comme présentation théâtrale et comme production plastique parmi les artefacts culturels ${ }^{31}$. Cette analyse de la prise de la Bastille est devenue rapidement un exemple modèle d'une démarche joignant deux stratégies : l'exploration de l'histoire de la culture et de la mentalité de la Révolution française s'accompagne d'une vue d'ensemble sur les effets culturels de ces symboles et mythes en Allemagne ${ }^{32}$. Dans une perspective identique, mais associée à l'histoire des concepts ${ }^{33}$, le Handbuch politisch-sozialer Grundbegriffe in Frankreich 1680-1820 (Dictionnaire des concepts politiques et sociaux fondamentaux en France de 1680 à 1820) publié en fascicules depuis 1985 , concrétise le développement et la différentiation de la perspective sémantique attestée dans les catégories de la science et la politique tant sous l'Ancien Régime que dans la Révolution ${ }^{34}$.

28 Parallèlement, Reichardt et de nombreux collaborateurs reconstruisent la "bibliothèque allemande de traduction des textes de la Révolution française " [Deutschfranzösische Übersetzungsbibliothek ${ }^{35}$. Recherches sur la sémantique historique, sur les structures narratives et sur le choix des textes à traduire permettent ainsi de reconstruire "l'image » de l'événement en France qui se relie à des figures ou concepts symboliques correspondants en Allemagne. Reichardt en conclut qu'une comparaison entre les actions d'État les plus importantes, le radicalisme politique des Jacobins en France et la courbe beaucoup plus à plat des insurrections et changements réformateurs en Allemagne, ne modifie pas le positionnement réciproque des deux espaces culturels liées par un courant ample d'informations. "La Révolution française » avait plutôt « dans l'espace culturel allemand son effet de masse le plus grand [...] sur le plan d'un public qui s'était politisé de manière subliminale ${ }^{36} \%$. Ainsi, à la fin de la décade révolutionnaire, le vocabulaire, les médias et l'horizon des changements désirables dans les deux pays sont largement semblables.

29 Ce changement partiel de perspective peut être atteint parce que la recherche du transfert culturel utilise de manière conséquente aussi bien des sources documentaires françaises que des documentations allemandes, ce qui démontre la présence d'une histoire des échanges culturels entre ces deux pays en matière archivistique ${ }^{37}$.

30 Le projet d'une étude approfondie des relations culturelles et intellectuelles entre la France et l'Allemagne au temps de la Révolution se fonde alors plus particulièrement sur trois méthodes :

31 - la reconstruction de toute la chaîne du transfert entre les deux cultures, de ses lieux de production jusqu'au moment de sa perception et de son usage, y compris la question importante des intermédiaires et des médias ;

32 - la combinaison du comparatisme déjà connu, et d'une histoire sociale aptes à compter et modeler les deux entités prises en compte avec une perspective culturaliste des perceptions, des adaptations intellectuelles et des transformations d'après les genres et médias utilisés ;

33 - la combinaison des études de cas avec une sérialisation des sources pour pouvoir se consacrer non seulement aux questions des métamorphoses culturelles du procès de 
transfert reconstructible seulement une à une, mais encore à la représentativité de ces processus. De ce besoin de sérialisation ressort nécessairement la considération des sources qui sont accessibles à un tel procédé, comme la presse, les collections de gravures et caricatures ou les archives d'autorités centrales intermédiaires, de même que les maisons d'édition ou les rédactions des périodiques et collections ${ }^{38}$.

Il ne s'agit donc pas d'une innovation qui refuse et met à part les méthodes jusqu'ici en usage, mais plus exactement d'une complexification croissante qui nécessite le recours au travail collectif et aux nouveaux supports médiatiques [en particulier les banques de données électroniques ${ }^{30}$ ] dans le but de dépasser les limites inhérentes au travail individuel.

II - Des résultats divers et nombreux

35 Ainsi, au cours des discussions sur le transfert culturel franco-allemand, les instruments méthodologiques s'améliorent au point de permettre aux résultats obtenus d'acquérir une représentativité de plus en plus grande. Dans le même temps, la recherche de détail permet de prendre conscience de l'asymétrie des espaces culturels et donne la possibilité à l'ancienne histoire régionale d'ouvrir aussi des chantiers sur des régions particulières, et non seulement sur l'ensemble de l'espace allemand. Cette dernière solution, qui multiplie les résultats utiles à l'histoire de la politique ou des structures sociales, ne suffit cependant pas à l'histoire culturelle. Nous constatons que la sensibilité aux offres étrangères est variable et se déploie sur des temporalités différentes dans les territoires allemands. Les espaces-frontières sont les plus intéressants pour les historiens. Il n'est donc guère étonnant de constater dans la région du Rhin une intensité particulière des processus de transfert ${ }^{40}$.

En regardant de plus près, la France n'apparaît pas si monolithique que la tradition du centralisme jacobin veut nous le faire croire. Nous trouvons des régions de communication dense avec l'étranger comme l'étude de Michel Espagne sur les commerçants du Bordeaux baltique le montre ${ }^{41}$. Il s'agit de communautés qui - par leurs activités économiques et sociales et leurs traits culturels - ont des relations intenses avec les grandes villes de commerce de la mer Baltique et du Nord. De son côté, Fred Schrader examine dans une vaste étude les structures de sociabilité de Hambourg et de Bordeaux; il étudie parmi des relations identiques du corps de commerçants, et bien plus intenses que l'intégration dans la culture locale correspondante ${ }^{42}$.

37 Il en résulte deux propositions pour la formation de notions et de concepts dans la recherche du transfert culturel. À la différence du concept de région utilisé jusqu'ici dans l'historiographie et qui considère seulement l'effet intérieur de l'intégration sociale et culturelle dans un territoire cohérent, le concept de " région européenne » se réfère au fait que les territoires tentent de conquérir leur modernité et leur dynamique par le moyen d'une réception particulièrement intense de l' "étranger ", d'un développement de la relation à l'autre. À ce titre, ils mobilisent la qualité particulière de leurs habitants par le biais de relations permanentes et culturellement utilisables par des régions étrangères ${ }^{43}$. Cette fonction d'intermédiaire ne se réfère pas qu'aux traducteurs, éditeurs, exportateurs ou importateurs singuliers du savoir technologique, mais aussi à des groupes sociaux entiers qui se distinguent assez souvent des autres par des caractéristiques confessionnelles (minorités religieuses) ou professionnelles (commerçants et prêteurs d'argent). À partir de leur fonction médiatrice durable et leur contact permanent à l'étranger, ces groupes développent - en dépit de leurs efforts 
de s'assimiler à une culture à laquelle ils restaient étrangers souvent pendant plusieurs générations - une « identité hybride » qui se révèle être particulièrement productive ${ }^{44}$. Katharina Middell traite ainsi de l'exemple des huguenots en Saxe où s'affirme pleinement cette identité polyvalente qui conserve longtemps les caractéristiques de deux cultures ${ }^{45}$. Par contre, les études entreprises par Thomas Höpel traitant des émigrés français en Prusse et Saxe montrent un large refus du rôle de l'intermédiaire interculturel ${ }^{46}$. La plupart des émigrés de la Révolution restent complètement attachés à leur ancien système de rapports culturels. Seuls font exception ces Français invités à rester dans les territoires allemands à cause de leurs capacités professionnelles ou artistiques : ils ont exercé là une fonction essentielle en contribuant à la modernisation de la culture d'accueil. L'histoire de l'échec du processus de transfert culturel en ce qui concerne les émigrés présente encore un côté intéressant. L'affluence massive d'étrangers (et qui, de plus, voulaient encore jouer un rôle militaire autonome avec leurs corps militaires), au moment où il s'agit bien d'éviter la confrontation et la déstabilisation entre les États, pousse à la redéfinition de la constellation. La politique prussienne soucieuse d'enregistrer les immigrants, de contrôler leur séjour, de fermer aux étrangers certains territoires et d'obtenir la continuation de leur voyage, ne se fonde pas seulement sur des stéréotypes et images «nationaux » d'ennemis de plus en plus fixes. En effet cette pratique d'administration, si imparfaite qu'elle apparaisse et si différente en soit l'utilisation dans les diverses provinces de l'État prussien, donne d'une certaine manière naissance à la notion moderne d' « étranger » défini et contrôlé par l'État et isolé culturellement du reste de la population ${ }^{47}$. Paradoxalement ce procédé s'exerce à l'exemple des émigrés qui ne sont pas regardés comme faisant partie des « Français de nation » (" Nationalfranzosen »).

Se pose alors la question de savoir quelle place la décennie révolutionnaire peut occuper dans une périodisation des transferts culturels franco-allemands. Tout jugement définitif est ici prématuré. Mais il existe plusieurs points de repère dans l'histoire des intermédiaires, celui des images de l'autre et des stéréotypes de l'« ennemi national », et enfin dans l'histoire des rapports de l'État avec les transferts culturels, qui confirment l'hypothèse de la naissance d'une constellation nouvelle dans la deuxième moitié des années quatre-vingt-dix. Le paradigme national devient de plus en plus déterminant dans l'analyse des transferts. Bien sûr, la concurrence des territoires particuliers pour l'obtention du rayonnement politique et culturel le plus fort ne disparaît pas en Allemagne. C'est pourquoi la réception de modèles de l'étranger est une tentation permanente. Mais à côté de la pratique de l'ouverture aux éléments étrangers, nous trouvons aussi la pratique de la séparation ou de la délimitation culturelle nationale. Le moment correspondant au niveau économique est l'effondrement des réseaux européens des négociants qui avaient depuis des décennies lié le continent par les flux des marchandises et leurs crédits réciproques. Les catégories de l'économie nationale et du protectionnisme les remplacent, selon une autre variante de la délimitation de l'étranger et peut-être de la supériorité technologique. Ce qui ne veut pas dire que le protectionnisme n'a pas eu une fonction essentielle dans le déploiement du marché intérieur et l'encouragement de l'industrialisation, ni que les processus de transferts culturels, après le cosmopolitisme de l'heureux XVIII ${ }^{e}$ siècle, soient paralysés dans la nuit d'un xIX siècle nationaliste. Bien au contraire. La densité croissante des communications et la consolidation des cultures nationales font que la période de la Révolution française à la Première Guerre mondiale devient le siècle élargi des transferts culturels par excellence ${ }^{48}$. 
Pourtant nous constatons une différence sérieuse, dans la mesure où la culture d'accueil aussi bien que la culture de départ est désormais une culture nationale, développée comme telle dans sa définition, sa structure et son insertion dans un cadre territorial. Les cultures régionales prennent ainsi une place subordonnée dans la hiérarchie de la conscience nationale; elles ont été cachées par les éléments nationalisants. Il faut donc peu à peu les "tirer à la surface »": un procédé contre lequel une histoire souvent encore attachée au paradigme national se défend de toutes ses forces en dénonçant les tentatives déjà mentionnées de provincialisme, alors que le reproche de provincialisme devrait s'adresser en priorité à l'histoire régionale (Landesgeschichte) traditionnelle ${ }^{49}$, qui se refuse à reconnaître et à étudier les rapports transnationaux des régions ${ }^{50}$. L'exemple des territoires allemands dans la Révolution de 1848-1849 montre cependant que les contextes et les expériences antérieures à l'existence de l'État national ont pu être ranimés au XIX siècle. Même dans la seconde moitié $\mathrm{du} \mathrm{XIX}^{\mathrm{e}}$ siècle l'originalité des rapports européens des régions allemandes demeure attestée ${ }^{51}$.

Les recherches sur les transferts culturels se sont lentement déplacées des études germaniques en France au terrain proprement dit de l'historiographie allemande. En passant ainsi dans un autre contexte de traditions et discussions, la perspective du transfert s'est enrichie entre autre par l'intérêt porté au rapport entre cultures nationale et régionale, entre histoire sociale et histoire culturelle où enfin entrent la méthode individualisante (Historismus) et la comparaison sociale, dont les enjeux suscitent depuis longtemps et encore de nos jours des controverses entre les historiens allemands. Dans le même temps, la sémantique historique et l'histoire de la diffusion de l'écrit (presse périodique, littérature, etc.) ont gagné en importance. De là résulte un projet scientifique important, qui s'occupe de la question de savoir si telle ou telle réflexion sur la Révolution française est connue en Allemagne et dans quelles couches sociales au cours des années quatre-vingt-dix. Cette étude représente un travail de base pour les études du transfert culturel, parce qu'elle donne la possibilité d'arriver au meilleur niveau de l'étude des relations franco-allemandes par le moyen de nouveaux documents tirés des archives et de méthodes novatrices appliquées à ces sources. Ainsi ont été sélectionnés comme indicateurs de la diffusion du savoir concernant les arguments, les mythes et les images courants en France, les traductions de textes français (des monographies et des articles de journaux) de la Révolution française parus en Allemagne. L'hypothèse de fond de ce projet consiste dans l'attribution à ces traductions d'une valeur explicative, dans la mesure où nous pouvons en étudier la diffusion sur le marché littéraire, voire même effectuer un sondage statistique des traductions et de leurs objets pour conclure à l'intérêt du public allemand.

41 La reconstitution de la "bibliothèque des traductions » fournit 16000 traductions allemandes des textes français entre 1770 et 1815 , dont le poids quantitatif porte nettement sur la période révolutionnaire. À partir d'une telle masse de données, nous pouvons tirer des conclusions représentatives sur les formes et les sujets de l'intérêt allemand à la Révolution française. Dès 1789 , l'article de journal est perçu comme le médium le plus opératoire, il prend de l'avance sur le livre, moins actuel et dont la traduction est de plus longue durée ${ }^{52}$. Déjà, d'après des recherches anciennes, nous savions que la réception allemande de la France avait été soumise à une politisation énorme. Plus précisément au plan quantitatif, avec 227 titres parus sur des thèmes explicitement politiques entre 1770 et 1789, le nombre croît à 1997 titres dans la 
décennie suivant la prise de la Bastille. La réception des sciences, de la philosophie et de la religion perd sa position d'avant-garde, bien que l'attention aux nouveaux résultats des sciences en France reste relativement élevée en Allemagne. Dans le domaine de l'art et de la culture, il s'opère un déplacement des formes anciennes au profit des romans et de l'opéra.

De la Révolution à l'Empire, l'intérêt se déplace de la politique intérieure aux affaires de l'extérieur, l'attention aux sciences continue à diminuer en pourcentage tout en restant élevée. On voit très bien ${ }^{53}$ comment le besoin de s'approprier des nouvelles de la France est soumis à un changement remarquable des formes et des sujets. Des intérêts établis - comme par exemple la littérature catholique du tournant $\mathrm{XVII}^{\mathrm{e}}$-XVIII ${ }^{\mathrm{e}}$ siècle, qui, durant les années 1780, était encore représentée dans la "bibliothèque des traductions » avec de nombreuses monographies volumineuses ${ }^{54}$ - n'ont plus trouvé de traducteurs, ni d'éditeurs ou d'acheteurs. Pour autant, une attention grandissante pour les ouvrages philosophiques issus des Lumières n'apparaît pas. Contrairement à toute attente, les Lumières françaises dans leur forme politisée ne se manifestent guère dans la "bibliothèque des traductions ", à rebours des recherches intenses sur l'influence des Lumières en Europe. Une explication se trouverait dans le fait que ces œuvres étaient suffisamment accessibles en langue française ${ }^{55}$ pour les lecteurs francophones. Mais il en résulte immédiatement une question : comment les textes politiques de la Révolution ont-ils pu avoir des effets dans un contexte où les couches moyennes de la bourgeoisie d'Ancien Régime n'avaient pas accès, y compris sous une forme rudimentaire, à leur fondement philosophique, et les parties alphabétisées du menu peuple ne connaissaient pas non plus le corpus de textes qualifiés de "grub street style » de la pornographie politique par Robert Darnton et d'autres chercheurs? Quoi qu'il en soit, il demeure que le public allemand est pris par la même fièvre des romans que celle que la France a connu dans la dernière phase des Lumières avant 1789. Ce qui signifie que le roman, souvent apolitique mais pénétré d'un rousseauisme trivial, n'a guère préparé la politisation du XVIII ${ }^{e}$ siècle finissant, mais l'a accompagnée et pour des parties du public l'a peut-être remplacée ${ }^{56}$. Nous en concluons donc que le transfert de textes de la Révolution française s'est opéré dans un contexte fort différent. Ainsi les travaux dont nous disposons actuellement ne répondent pas encore complètement aux conséquences de cette réception dans un contexte autre, mais les données statistiques permettent après tout de poser des questions par-delà les simples spéculations.

$\mathrm{Au}$ sein de la liste des auteurs les plus traduits ${ }^{57}$, il convient de faire une différence entre les monographies et les articles, car seul Voltaire - l'auteur français le plus souvent traduit en Allemagne (321 textes, dont 216 monographies, surtout des pièces de théâtre) - et Mercier figurent entre 1770 et 1815 parmi les dix premiers sur les deux listes. Une fois de plus, nous constatons que la transformation des textes traduits, par le passage de gros livres à des articles, marque un changement radical. Désormais, d'autres auteurs s'offrent aussi pour une traduction. Louis Sébastien Mercier, dont le style fragmentaire s'adapte au mode de réception dominant après 1789 , en est l'exemple type (68 traductions d'articles, 90 traductions de monographies). Napoléon Bonaparte est l'auteur le plus traduit à travers ses déclarations et de petits articles (217 traductions d'articles), qui semblent garantir aux traducteurs de même qu'aux éditeurs un large écho auprès du public. Au sein des politiciens les plus actifs de la Révolution on trouve, parmi les plus traduits, Roederer, Carnot, Robespierre, Jean Gabriel Peltier, Mirabeau, Dumouriez, Necker, Condorcet, Brissot et Louis XVI, liste d'auteurs 
majoritairement modérés, mais de toute façon représentatifs de l'aspect de la Révolution qui touche à la manière proprement allemande d'appréhender la compréhension des événements français, surtout centrée sur les décisions relatives à la forme politique de la société et celles qui concernent la politique extérieure et militaire de la France.

Dans le même temps, le marché des traductions devient de plus en plus professionnalisé. À côté d'une foule immense d'amateurs ne traduisant en allemand qu'un ou deux textes, il se forme un groupe de cinquante personnes qui traduisent un grand nombre de textes. Dans ce groupe se distinguent quatre traducteurs (Faber, Gotter, Huber ${ }^{58}$ et Pfeffel) : à eux seuls, ils ont traduit plus de 400 textes ${ }^{59}$. Des véritables "fabriques de traduction" ${ }^{60}$ se forment donc, et plusieurs journaux importants commencent à se faire concurrence dans l'obtention des informations les plus actuelles et précises sur la France ${ }^{61}$ en donnant des informations " primaires » qui souvent se révèlent être des collections de traductions ou de comptes-rendus détaillés de textes français ${ }^{62}$.

Ces indications montrent déjà en quoi l'analyse statistique des traductions en allemand a pu stimuler plusieurs champs, s'actualisant au sein des recherches antérieures sur l'histoire des médias et des intermédiaires culturels, le tout convergeant vers une approche nouvelle.

III - Une problématique ouverte

La thèse d'habilitation récemment parue de l'historien fribourgeois Erich Pelzer, soutenue en 1994, et visiblement entreprise en parallèle au projet de Reichardt et Lüsebrink ${ }^{63}$, manifeste cependant des problèmes résultant des nouvelles méthodes. Pelzer, qui tente également de reconstituer le corpus d'ensemble des traductions allemandes, s'en tient à la période 1789-1799. Il a fouillé les bibliographies, les annonces des éditeurs, les catalogues des foires du livre et les listes de la censure ainsi que les catalogues des grandes bibliothèques pour arriver à 2000 traductions de textes historico-politiques ${ }^{64}$ (un nombre clairement inférieur à celui recensé par Lüsebrink et Reichardt même si nous regardons seulement les années étudiées en commun par les deux travaux). L'explication principale est liée au fait que Pelzer cherche des critères rendant compte du contenu de sa collection de textes, à savoir l'attribut de la spécificité historico-politique, tandis que Lüsebrink et Reichardt prennent en compte la totalité des traductions du français. En regardant les titres indiqués par Pelzer, nous remarquons alors la surreprésentation des monographies, tandis que le nombre des articles est relativement petit malgré l'étude faite aussi sur les principaux journaux ${ }^{65}$. Mais en même temps, Pelzer découvre pour quelques auteurs des indications plus précises que celles de Lüsebrink et Reichardt. Ayant publié en annexe la liste des traductions qu'il a découvertes, ajoutée à la liste également publiée des 16000 titres relevés par Lüsebrink, Reichardt et ses collaborateurs, une comparaison précise est possible, en particulier à l'aide d'un CD-ROM comprenant déjà la seconde liste. Cependant la non-coïncidence troublante des deux recensements impose un commentaire: l'exactitude, vis-à-vis des lecteurs, des tableaux et des diagrammes portant sur un très grand nombre de cas est fonction, plus encore que dans les recherches anciennes, de la publication précise des méthodes et des documents de base, dans la mesure où les lecteurs n'ont plus les moyens personnels de reconstituer la genèse de la composition des données. Avec l'explosion des frais de production surtout pour la publication des données de base et le nombre restreint des experts, il devient de 
plus en plus difficile de répondre à cette demande de visibilité de données quantitatives qui s'impose d'un autre côté par le progrès des méthodes. Rester au seul niveau de la publication des résultats sous forme de données quantitatives, c'est prendre le risque de s'interdire toute opération de vérification par la communauté des chercheurs dans son ensemble.

La situation est étrange: deux études arrivent à des résultats différents tout en revendiquant chacune la préséance en matière d'analyse exhaustive. Reste, et c'est l'avantage de cette situation, que la littérature parue en Allemagne sur la Révolution française est désormais connue dans le détail, que ces deux études parallèles fournissent des bibliographies utiles dont on peut espérer qu'elles seront rapidement accessibles à tout le monde.

Pelzer confirme l'affinité du public allemand avec les auteurs girondins, une fois que le grand intérêt initial pour les monarchiens s'avère tari, c'est-à-dire au moment où les monarchiens tombent dans l'insignifiance. Sa remarque sur l'orientation politique majoritaire des auteurs-journalistes allemands du côté des Girondins (Oelsner, Cramer, Kerner, Reinhard, Archenholz) confirme les résultats des recherches plus anciennes et montre en même temps le rôle central des intermédiaires dans les processus de transfert ${ }^{66}$. Son choix conséquent de quelques-uns de ces intermédiaires paraît cependant sans motivation suffisante et retombe ainsi dans une historiographie personnalisante, alors que l'histoire des transferts culturels en se fondant sur des méthodes de l'histoire sociale veut justement la dépasser. Là où il donne les motifs de l'adhésion, pour lui primordiale, des intellectuels allemands aux opinions girondines, modèles d'explication produits autrefois par les théories des " caractères nationaux ", il est difficile de suivre ses arguments. D'après lui, les intellectuels allemands, introvertis et donc trop occupés par leurs intérêts et idées politiques abstraits, se voulaient éloignés des révolutionnaires français, hommes d'action prêts à prendre des décisions immédiates ${ }^{67}$. Une telle interprétation reprend sans esprit critique des anciens préjugés qui se trouvent attestés dans les sources et documents eux-mêmes et méritent plus qu'une simple répétition. La thèse centrale de Pelzer est donc la suivante : les intellectuels allemands auraient traduit, publié, acheté et lu avec prédilection les textes des girondins parce qu'ils pouvaient, ainsi drapés dans les costumes des révolutionnaires français mis en échec tels Louvet, Riouffe, Pétion et Sieyès, mieux légitimer leur propre manque d'action. Elle repose sur une bibliographie discutable, mais elle montre la liaison des processus de transferts culturels entre l'Allemagne et la France et précise les motifs du transfert dans la recherche des orientations et des modes d'expression politiques. Ainsi l'idée de partir de la Révolution elle-même et de sonder ce qui est vraiment "arrivé » dans l'espace culturel allemand ${ }^{68}$ introduit, comme nous l'avons déjà souligné, un changement très productif dans la pratique de la recherche. L'instrument herméneutique du concept de transfert culturel est cependant insuffisamment utilisé s'il se limite au constat du nombre et de la répartition thématique des traductions. Il reste à poser des questions sur les motivations (qui transforment le texte d'origine) et les conséquences (qui traduisent le transfert en articulation politique) de cette réception.

$\mathrm{Au}$ cours des dernières années, l'historiographie allemande a produit là-dessus des contributions intéressantes qui intègrent le nationalisme naissant dans le schéma de l'interprétation nouvelle de la Révolution française en tant que révolution culturelle ${ }^{69}$. La re-définition en partie novatrice de la Révolution française après la conjonction de 
l'histoire des mentalités, l'histoire sociale et culturelle de idées, l'analyse des langages, des discours et des usages des mots s'ouvre bien au-delà de l'histoire usuellement plus étroite des idées. Ce développement a son prix, surtout au moment où l'on quitte le champ de l'historiographie spécialisée de la Révolution pour se confronter à d'inévitables vulgarisations, sous forme d'un résumé globalisant de résultats différenciés dans d'autres champs de recherche. Ce prix à payer consiste souvent dans une négligence des résultats de l'histoire sociale et économique, certes au profit d'une histoire politique renouvelée, dans laquelle on peut plus facilement reconstruire les relations entre les pratiques discursives et les actions politiques. Ainsi le passage à la nouvelle histoire culturelle s'associe plus facilement à la renaissance de l'analyse du politique. Nous en avons plusieurs exemples.

50 Nous regardons alors les structures sociales et les intérêts économiques comme des chaînes dont l'histoire de la culture devrait nous libérer de manière à révoquer la dictature $\mathrm{du}$ modèle weberien, répandue de façon particulièrement stable en Allemagne de l'Ouest ${ }^{70}$. De cette constellation spécifique de l'historiographie allemande résulte un "découpage socio-professionnel " particulièrement marqué ${ }^{71}$, dans la mesure où les modèles interdisciplinaires au sein des divers courants de l'histoire sont moins accessible qu'en France, surtout pour des raisons intellectuelles et institutionnelles. Cependant, nous pensons avoir déjà montré que l'historiographie allemande se détache progressivement, mais sur le ton de la polémique, des orientations longtemps dominantes : elle construit des catégories aptes à s'insérer dans la nouvelle histoire de la culture et redécouvre des traditions ensevelies de sa propre préhistoire. Des thèmes comme l'identité nationale et les facteurs qui la stabilisent tels la mémoire et le mythe ${ }^{72}$ sont désormais au centre de la réflexion, et non relégués en fin de parcours, se rapprochant ainsi des recherches traditionnellement intenses sur l'histoire la plus récente des identités démultipliées par la modernité.

51 En matière d'histoire franco-allemande de la fin XVIII ${ }^{e}$ et du XIX ${ }^{e}$ siècles, ce sont surtout les travaux de Michael Jeismann ${ }^{73}$ et de Andreas Dörner ${ }^{74}$ qui ont renouvelé la perspective. Jeismann montre dans son étude sur la longue durée - du début des guerres révolutionnaires jusqu'à la fin de la Première Guerre mondiale - comment l'ennemi est devenu un élément constitutif de l'identité nationale. Cet ennemi fait obstacle à l'accomplissement de la mission située au fondement du projet national, et en même temps incarne, dans sa défaite, la possibilité de pouvoir atteindre ce but. Sans l'ennemi, le caractère prometteur et presque religieux de la nation perd de sa crédibilité. Les notions, les imaginaires et les oppositions établis à la fin du XVIII et au début du XIX $\mathrm{X}^{e}$ siècle s'avèrent ainsi d'une persistance étonnante, survivent par les répétitions réitérées des changements de courte durée intervenues dans la situation politique. Sous le manteau de l'expérience historique, la stylisation mythique prouve toujours son utilité.

52 Mais les deux façons de construire la mythologie nationale diffèrent en France et en Allemagne. À l'opposé de la justification universaliste de la supériorité du projet national en France, l'Allemagne nous renvoie au caractère moral et chrétien de la nation ${ }^{75}$. Le travail de Jeismann corrige plusieurs éléments de la recherche sur les nationalismes dans la mesure où il analyse, en lieu et place de typologies abstraites des nations et nationalismes, leur genèse et la réciprocité des processus de "l'invention » des traditions ${ }^{76}$. 
53 Il en résulte, en matière d'étude de l'époque révolutionnaire dans la perspective des transferts culturels, des questions sur l'articulation de l'ensemble des processus qui mènent à l'élaboration et la diffusion des projets nationaux. Ici le livre de Andreas Dörner, qui se consacre à une théorie du mythe dans la culture politique et, au plan empirique, à l'étude du topos de la Hermannsschlacht, se rattache directement aux travaux mentionnés plus haut sur la politisation fondamentale opérée par la Révolution dans les territoires allemands: "La carte cognitive du politique, sans être véritablement mise en question sous l'Ancien Régime, a vécu avec l'apparition de nouveaux groupes d'acteurs des transformations énormes; tout l'espace des signes culturels se trouve fondamentalement politisé ; et on constate une intensification et une démocratisation accélérée de la structure communicative de la société ${ }^{77}$. " D'une dimension jusqu'alors inconnue, la politique des symboles (ou politique symbolique) devient l'instrument de l'intégration sociale et de la délimitation de l'identité nationale des autres identifications. La rupture peut-être la plus radicale avec les usages connus $\mathrm{du}$ politique est - justement dans la perception allemande - la décapitation de Louis XVI ${ }^{78}$. Désormais les ordres du sens (Sinnordnungen) ne sont plus éternels et préexistants mais sont compris comme ce que l'on peut et que l'on doit construire de manière active. Ainsi le sens politique ouvre au pluralisme: il importe donc de le délimiter s'il doit conserver sa fonction de garant de la sûreté en matière d'action sociale. La mise en scène esthétique et la politique des symboles deviennent de plus en plus nécessaires dans le contexte de la nouvelle culture politique ${ }^{79}$ dégagée par la Révolution, même si elles ne sont pas l'invention de la Révolution elle-même et existaient déjà sous l'Ancien Régime. Deux modèles de mise en scène s'établissent. D'abord les élites transforment assez vite l'histoire événementielle «chaotique » et contradictoire en une narration cohérente reposant sur une téléologie qui parcourt la description des étapes de la Révolution : nous y retrouvons les explications causales élaborées depuis la convocation des États généraux. Indépendamment des divergences doctrinales entre les divers groupes dans la Révolution, ils utilisent tous ce modèle, dont les structures narratives et les fonctions dans la culture commémorative sont maintenant bien connues. En second lieu, la continuité avec le passé le plus récent est dénoncée. L'invention d'un Ancien Régime qui n'a rien de commun avec la société révolutionnaire, voire même s'y oppose point par point, est à l'ordre du jour. Ainsi se construit une préhistoire à la Révolution en référence à la république romaine, voire même à l'Empire romain ${ }^{80}$, et cette préhistoire permet la démultiplication des thèmes de la représentation iconographique ${ }^{81}$ et l'introduction des fêtes et des rituels. Ces formes de politique symbolique suscitées par la formation initiale du mythe et de sa narrativité ont été également utilisées dans la répétition et l'exercice collectif du mythe, par lequel chaque individu peut trouver sa place dans la société nouvellement créée. Une telle invention est possible parce que les formes de diffusion du mythe s'adressent à des milieux culturels divers et se transforment peu dans le trajet de la répétition spontanée à une sorte de liturgie.

Enfin, l'ensemble de ces nouvelles études sur l'histoire de la culture révolutionnaire en France et sur les transferts culturels franco-allemands influent et modifient les arguments de l'histoire de l'Allemagne. Andreas Dörner remarque que les réformes (militaire, municipale, agraire et du système d'éducation) en Prusse après 1806 proviennent des réactions des élites aux causes bien connues des victoires des troupes napoléoniennes mais dont l'impact ne pouvait être que de longue durée. C'est pourquoi ces réformes sont demeurées sans effet immédiat dans le tournant de la guerre. Par 
contre se manifeste là une construction prospective du sens politique à l'aide d'un mythe politique alternatif, devenu progressivement le médium de la mobilisation du peuple et la condition pour une contre-attaque couronnée de succès dans la guerre populaire ${ }^{82}$. Scharnhorst, Clausewitz et d'autres ont déjà reconnu en 1793-1797 que le soldat patriote et citoyen, soldat politisé par excellence, est le fondement des succès français alors que les anciennes troupes mercenaires, à cause de leurs règles d'enrôlement, ne disposent pas d'un tel modèle du soldat-citoyen. Tandis que la France avait appris sa leçon de la bataille de Roßbach en 1756 (et en pouvait faire preuve dans la guerre d'indépendance en Amérique), la Prusse en tire les conséquences beaucoup plus tard. Le point de départ est cependant semblable : le but ne consiste plus à rendre la guerre plus humaine comme ce fut le cas après les guerres de religion mais la politisation de la guerre est le but lui-même; qui plus est la moralisation et la totalisation de la guerre deviennent l'instrument et la conséquence de cette stratégie ${ }^{83}$. L'idée que l'adversaire est l'ennemi de toute l'humanité, donc doit être anéanti, prend la place de la vision traditionnelle de l'opposition ami-ennemi. Ernst Moritz Arndt, un des idéologues d'une telle propagande, est aussi un observateur très attentif de la "politique (française) des mythes " ${ }^{84}$. De même, la transformation des mentalités dans les États allemands ne peut pas être seulement comprise comme le refus de l'occupation française, mais doit être aussi perçue comme l'imitation des principes constructeurs de la politique française réadaptée, dans un processus complexe de transfert culturel, aux besoins des Allemands.

D'après le travail de Andreas Dörner on peut suivre trois processus distincts :

- la création d'un acteur collectif "peuple» ou «nation» en direction d'une population soucieuse de se définir un rôle dans la société nouvelle ;

- la concrétisation de ces acteurs dans une structure narrative mythique qui répand une symbolique effective et engendre par son pathos la volonté d'être prêt à l'action ;

- le glissement des références culturelles à travers une campagne dé-civilisatrice, dans laquelle au lieu de l'humanité et de l'amour du prochain, les hommes ont recours à la haine, la violence et la revanche.

Le discours sur la nation est antérieur à ces processus, en Allemagne aussi. Il réagit d'abord - comme dans le cas de Herder - en identifiant la langue et l'histoire, la littérature et les arts communs aux besoins d'identification d'une société des États en décomposition. La politisation d'un discours préexistant n'est que le produit des temps des guerres antinapoléoniennes («Befreiungskriege»), porté par des formes d'organisations diverses ${ }^{85}$.

57 Le rapport aux processus de transferts culturels des années quatre-vingt-dix saute ainsi aux yeux. Des techniques de politisation et mobilisation de la population, déjà éprouvées en France, sont reprises, mais adaptées à un contexte différent. Les besoins de la culture d'accueil dirigent le choix et l'emploi de ce qui doit être reçu. Les sociétés voisines sont contraintes de réagir face à un tel défi de politisation de la symbolique et de l'imaginaire par la Révolution, au plus tard au moment où elles sont confrontées aux succès de cette mobilisation. Mais en même temps il s'agit de rendre compte des caractéristiques spécifiques d'un territoire où il n'y a pas d'État uni et où l'ancienne société n'a pas été détruite. La possibilité d'affirmer une discontinuité radicale par rapport à l'ancienne société et aux élites n'existe donc pas. D'un élément à l'autre se construit une mixité dynamique de formes, rituels, mythes modernes et traditionnels. La nation devient, selon le modèle français, un sujet agissant. Il est désormais possible 
de lui donner une histoire rattachée directement au sens de son déploiement futur. L'honneur - perdu mais à reconquérir - devient l'élément central d'une structure narrative qui ne se représente pas comme la simple prolongation du présent dans un avenir universel, mais c'est l'idée d'une délivrance individuelle du rôle des martyrs qui donne à la nation du sens et de la signification. À la différence de l'invention française, l'invention allemande de la nation ne connaît pas ce conflit frontal avec la tradition de l'Église et de la religion, elle peut au contraire recourir à la force mobilisatrice des narrations religieuses, surtout protestantes ${ }^{86}$.

Cependant l'histoire des transferts culturels franco-allemands ne peut pas être réduite à l'histoire de la genèse de la nation et de leur hostilité réciproque, même si cette histoire est très influente à long terme. Des recherches en cours s'occupent des articulations dans le domaine de l'économie, des sciences ou des arts. Elles débutent seulement, comme le montre la tentative récente d'écrire une synthèse de la Révolution française, du point de vue des recherches nouvelles ${ }^{87}$. Jusqu'à présent le territoire de l'histoire des transferts culturels ressemble plus à un lieu d'exploration par des chercheurs curieux d'un terrain peu connu qu'à un champ strictement délimité. À la différence d'autres paradigmes de l'historiographie, nous ne constatons pas encore de tentatives sérieuses d'institutionnalisation précédant une canonisation. C'est pourquoi tout bilan définitif reste prématuré, la liste des questions ouvertes demeure plus longue que celle des solutions. Et ce n'est pas la moindre des questions à poser, si l'on se demande pourquoi nous voulons écrire une histoire des aspects étrangers souvent cachés, mais tout à fait virulents dans l'histoire des communautés culturelles.

Il est vrai qu'il s'agit, avec le passage de l'ancienne histoire comparatiste à l'histoire des processus de transferts culturels, de la relève d'un paradigme par un nouveau paradigme. Mais il n'y a pas là occasion à «brûler des livres ». La critique suscitée par les recherches en terme de transfert s'est attachée à quelques déficits éclatants et dangereux de la comparaison antérieure. Mais, d'un autre côté, cette critique n'a rien de très nouveau. L'essai classique écrit par Marc Bloch dans les années vingt fournit déjà beaucoup de réflexions en la matière : les comparatistes ne le citent-ils pas comme une sorte de texte fondateur pour leur propre légitimation! À ce titre, le chercheur qui expose les limites du comparatisme ne fait pas autre chose que de faire resurgir ce que l'histoire comparatiste a en partie oublié ou écarté. Ainsi il importe d'éviter de jeter le bébé avec l'eau du bain. Les recherches récentes concernant les transferts culturels se basent sur les résultats de l'histoire sociale, soit en utilisant les catégories de la différenciation sociale dans l'analyse des intermédiaires culturels, soit en recourant aux structures sociales de la composition des sociétés de lecture, des académies et sociétés populaires. Le regard comparatiste sur les différences du niveau de la productivité, de l'infrastructure ou du degré du remplacement des rapports féodaux par des rapports capitalistes dans l'économie rurale à la fin du xviIIe siècle, n'est donc pas écarté, mais plutôt complété par la question de savoir dans quelle mesure il paraît essentiel aux yeux des contemporains, pour qu'ils puissent se voir dans des mondes divers et en tirer des manières d'agir politiquement. Ce procédé est bien plus un raffinement de questions plus anciennes que leur relève complète. Mais bien entendu, chaque époque de l'historiographie s'annonce dans un climat d'innovation. Une fois le calme rétabli sur le champ de bataille après le bruit du canon, nous pouvons calculer plus précisément les acquis et les pertes. 

tiens à remercier Marc Muylaert (Leipzig) et Jacques Guilhaumou (Aix-en-Provence) pour l'aide qu'ils m'ont apportée afin d'établir la version française de ce texte. Mes remerciements vont également à mes collègues du groupe de recherches sur le transfert culturel franco-saxon pour leurs commentaires toujours précieux.

\section{NOTES}

1.Pour plus d'indications sur les tendances les plus récentes vers une histoire culturelle dans l'historiographie allemande en général, voir M. MIDDELL, "Jalons bibliographiques. Les approches allemandes récentes de l'histoire culturelle”, Revue Germanique Internationale $\mathrm{n}^{\circ} 10,1998$, pp. 231-238.

2.M. vOVELLE (éd.), L'image de la Révolution française, 4 vols, Paris 1989; M. кOSSOK/ E. KROss (éds), 1789 - Weltwirkung einer großen Revolution, 2 vols, Berlin 1989.

3.J. GARBER, "Peripherie oder Zentrum ? Die europäische "Triarchie" (Deutschland, Frankreich, England) als transnationales Deutungssystem der Nationalgeschichte", dans: M. ESPAGNE/ M. WERNER (éds), Transferts. Les relations interculturelles dans l'espace franco-allemand (XVIII ${ }^{e}$ XIX $X^{e}$ siècle), Paris 1988, pp. 97-162.

4.Des bilans sont parus à l'occasion du bicentenaire: A. SAITTA (éd.), La storia della storiografia europea sulla rivoluzione francese (Relazioni Congresso maggio 1989), 3 vols, Rome 1990-1991; M. vovelle (éd.), Recherches sur la Révolution. Un bilan des travaux scientifiques du Bicentenaire, Paris 1991; le même (éd.), Les colloques du Bicentenaire. Répertoire des rencontres scientifiques nationales et internationales, Paris 1991; K. MIDDELL/ M. MIDDELL/ M. коSSOK/ M. voveLLE (éds), 200. Jahrestag der Französischen Revolution. Kritische Bilanz der Forschungen zum Bicentenaire, Leipzig 1992.

5.B. FAULENBACH, Ideologie des deutschen Weges. Die deutsche Geschichte in der Historiographie zwischen Kaiserreich und Nationalsozialismus, München, 1980.

6.Un premier essai de comparaison: M. MIDDELL, "Metaerzählungen: Vergleichende Revolutionsgeschichte und Sonderwegsthese", Berliner Debatte Initial, 9 (1998), n 5, pp. 59-75.

7.Pour la multiplication des variantes du comparatisme voir encore l'article classique de Marc Bloch de 1928. Moins critique en face des vieux modèles comparatistes des années 1960 se présente le résumé récent de H. KAELBLE, Der historische Vergleich. Eine Einführung zum 19. und 20. Jahrhundert, Frankfurt a. M., 1999 et H.-G. НАUPT/ J. КоскA (éds), Geschichte und Vergleich. Ansätze und Ergebnisse internationale vergleichender Geschichtsschreibung. Frankfurt a. M., 1996.

8.J. PAULMANN, "Internationaler Vergleich und interkultureller Transfer. Zwei Forschungsansätze zur europäishen Geschichte des 18. bis 20. Jahrhunderts", Historische Zeitschrift vol. 267, 1998, n 3, pp. 649-685.

9.Jakobinismus und Volksbewegung zur Zeit der Französischen Revolution. Dem Wirken Walter Markovs gewidmet (Sitzungsberichte der AdW in Berlin, 8 G 1990), Berlin, 1991; H. WILHARM, Politik und Geschichte. Jakobinismusforschung in Deutschland, 2 vols, Franfurt a. M./ Bern/ New York, 1984; W. KREUTZ, "Von der "deutschen" zur "europäischen" 
Perspektive. Neuerscheinungen zu den Auswirkungen der Französischen Revolution in Deutschland und der Habsburger Monarchie", Neue politische Literatur vol. 31, 1986, pp. 415-441.

10.Voir les études rassemblées dans: W. MARKov, Weltgeschichte im Revolutionsquadrat, publ. par M. Kossok, Berlin, 1979.

11.Au sein d'une bibliographie énorme, nous citerons surtout de H.-U. WEHLER, Deutsche Gesellschaftsgeschichte, dont 3 des 4 volumes prévus sont parus, et J. коскА (éd), Bürgertum im 19. Jahrhundert. Deutschland im europäischen Vergleich, München, 1988, 3 vols. Pour l'ensemble on consultera H.-U. WEHLER, Bibliographie zur neueren deutschen Sozialgeschichte, München, 1993.

12.Surtout les onze volumes des Studien über die Revolution, publ. par Manfred Kossok, Berlin, 1971-1989.

13.Comme exemple de cette ouverture vers une comparaison plus détaillée: H. BERDING/ E. FRANÇOIS/ H.-P. ULLMANN (éds), La Révolution, la France et l'Allemagne: deux modèles opposés de changement social ?, Paris, 1989.

14.Dans la perspective des recherches pionnières de Reinhart Koselleck, la "sémantique historique" concerne l'analyse des structures langagières des expériences historiques, de la sémantique des concepts au discours du vécu historique. $C f$. dans la traduction française, Le futur passé. Contribution à la sémantique des temps historiques, Paris, EHESS, 1990.

15.R. REICHARDT, "Die Französische Revolution als Maßstab des deutschen "Sonderwegs" ? “, dans: J. Voss (éd.), Deutschland und die Französische Revolution, München, 1983, pp. 323-327.

16.Quelques années plus tard encore son plaidoyer pour un comparatisme dans la perspective d'une histoire culturelle provoque des longues querelles au sein d'un colloque à Mayence: R. REICHARDT, "Die Französische Revolution und Deutschland Thesen für einen komparatistischen kulturhistorischen Neuansatz", dans: K. O. v. ARETIN/ K. HÄRTER (éds), Revolution und konservatives Beharren: das alte Reich und die Französische Revolution, Mainz, 1990, pp. 21-28.

17.R. REICHARDT, "Bevölkerung und Gesellschaft Frankreichs im 18. Jahrhundert. Neue Wege und Ergebnisse sozialhistorischer Forschung 1950-1976", Zeitschrift für historische Forschung 1977, n 4, pp. 231-249; du même, "Auf dem Weg zu einer Totalgeschichte des ländlichen Franreich”, Historiusche Zeitschrift vol. 224, 1977, pp. 635-654; du même, "Histoire des mentalités". Eine neue Dimension der Sozialgeschichte am Beispiel des französischen Ancien Régime", Internationales Archiv für Sozialgeschichte der Literatur 1978, pp. 130-166; du même, "Zu einer Sozialgeschichte der französischen Aufklärung. Ein Essay", Francia. Forschungen zur westeuropäischen Geschichte, vol. 5, 1978, pp. 231-249. 18.M. козSок, "Revolutionärer und reformerischer Weg beim Übergang vom Feudalismus zum Kapitalismus. Ein Diskussionsbeitrag”, Leipziger Beiträge zur vergleichenden Revolutionsforschung, vol. 16, 1986, pp. 6-39.

19.R. KOSELLECK/ R. REICHARDT (éds), Die Französische Revolution als Bruch des gesellschaftlichen Bewußtseins, München, 1988. Pour une évolution vers une interprétation culturelle et sociale de la Révolution voir aussi M. коssoK, "Realität und Utopie des Jakobinismus. Zur "heroischen Illusion" in der bürgerlichen Revolution", Zeitschrift für Geschichtswissenschaft 1986, n 5, pp. 415-426. 
20.R. REICHARDT, "Von der politisch-ideengeschichtlichen zur sozio-kulturellen Deutung der Französischen Revolution. Deutschsprachiges Schrifttum 1946-1988”, Geschichte und Gesellschaft 1989, n 1, pp. 115-146.

21.Voir les débats dans HAUPT/ КОСКА, op. cit. (note 7).

22.M. ESPAGNE, "sur les limites du comparatisme en histoire culturelle", Genèses n ${ }^{\circ} 17$, 1994, pp.112-121.

23.M. ESPAGNE/ M. WERNER, “Deutsch-französischer Kulturtransfer im 18. und 19.

Jahrhundert. Zu einem neuen interdisziplinären Forschungsprogramm des C.N.R.S”., Francia vol 13, 1985, pp. 502-510; id., "La construction d'un réference culturelle allemande en France. Genèse et histoire", Annales E.S.C., 1987, pp. 969-992; id., "Deutschfranzösischer Kulturtransfer als Forschungsgegenstand. Eine Problemskizze”, id. (éds), Transferts ... (note 3), pp. 11-34.

24.Id,. pp. 5 et 8.

25.K. MIDDELL/M. MIDDELL, "Migration als Forschungsfeld", Grenzgänge 5 (1998), pp. 6-23 et comme exemple de la recherche des migrations et des études sur la mémoire collective: K. RANCE, "L'identité collective des nobles français émigrés en Allemagne (1789-1815)", ibid., pp. 24-37 ; de la même, "L'émigration nobiliaire française en Allemagne : une "migration de maintien" 1789-1815", Genèses n 30, 1998, pp. 5-29 ; Th. HÖPEL/K. MIDDELL (éds), Réfugiés und Émigrés. Migration zwischen Frankreich und Deutschland im 18. Jahrhundert, (= Comparativ, nos 5/6), Leipzig, 1997 ; J. MONDOT/ J.-M. VALENTIN/ J. voss (éds), Deutsche in Frankreich - Franzosen in Deutschland 1715-1789. Institutionelle Verbindungen, soziale Gruppen, Stätten des Austausches, Sigmaringen, 1992.

26.F. BARBIER, L'empire du livre. Le livre imprimé et la construction de l'Allemagne contemporaine 1815-1914, Paris, 1995; H. JEANBLANC, Des Allemands dans l'industrie et le commerce du livre à Paris 1811-1870, Paris, 1994.

27.H. SIEGRIST et al., Europäische Konsumgeschichte, Frankfurt a. M., 1998.

28.E. POMMIER, “Christian Ludwig von Hagedorn und Roger de Piles”, dans M. ESPAGNE/ M. MIDDELL (éds), Von der Elbe bis an die Seine. Kulturtransfer zwischen Sachsen und Frankreich im 18. und 19. Jahrhundert, Leipzig, 1993, pp. 31-51.

29.M. ESPAGNE, "Französisch-sächsischer Kulturtransfer im 18. und 19. Jahrhundert. Eine Problemskizze" Comparativ 2 (1992), no 2, pp. 100-121. Voir aussi notre résumé des publications parues jusqu'en 1994: K. MIDDELL/M. MIDDELL, "Forschungen zum Kulturtransfer. Frankreich und Deutschland", Grenzgänge 1 (1994), no 2, pp. 107-122. 30.Pour une définition du symbole collectif spécifique à la culture dont il est né, et des éléments stéréotypes dépassant la qualité des signes culturelles de la langue pour intégrer des discours et des pratiques très divers de cette culture, voir: A. DRES/U. GERHARD/J. LINK, "Moderne Kolektivsymbolik. Eine diskurstheoretisch orientierte Einführung mit Auswahlbibliographie", in: Internationales Archiv für Sozialgeschichte der deutschen Literatur, numéro thématique 1, Tübingen, 1985, pp. 256-365.

31.H.-J. LÜSEBRINK/R. REICHARDT, Die "Bastille" Zur Symbolgeschichte von Herrschaft und Freiheit, Frankfurt a. M., 1990, particulièrement pp. 13 et suiv. et p. 260. Voir aussi les mêmes, "La "Bastille" dans l'imaginaire social de la France à la fin du Xviıe siècle (1774-1799)", Revue d'histoire moderne et contemporaine, n 30 (1983), pp. 196-234. 32.Pour le mythe de la Bastille: R. REICHARDT, "Deutsche Volksbewegungen im Zeichen des Pariser Bastillesturmes", in: H. BERDING (éd.), Soziale Unruhen in Deutschland während der Französischen Revolution, Göttingen, 1988, pp. 10-27; le même, "Bastillen in Deutschland? Gesellschaftliche Außenwirkungen der Französischen Revolution am 
Beispiel des Bastillesturmes", in: R. MELVILLE/C. SCHARF/M. VOGT/U. WENGENROTH (éds), Deutschland und Europa in der Neuzeit, Stuttgart, 1988, pp.419-467.

33.Dont R. KOSELLECK est le personnage principal au sein de la vaste entreprise $d u$ Geschichtliche Grundbegriffe: Historisches Lexikon zur politisch-sozialer Sprache in Deutschland (Les concepts fondamentaux en histoire: Dictionnaire historique du langage politicosocial en Allemagne) sous la direction de Otto BRUNNER, Werner CONZE et Reinhart KOSELLECK, Stuttgart, 1972-1996.

34.Fasc. 1-10 publ. par R. REICHARDT et E. SCHMITT, à partir du fascicule 11 par R. REICHARDT et H.-J. LÜSEBRINK.

35.Les premières explorations du corpus croissant de textes dans: R. REICHARDT, “" "Freymüthigkeit, doch kein Sans-Cülotismus...”. Transfer und Transformation der Französischen Revolution in Verdeutuschungen französischer Revolutionsschriften 1789-1799”, dans: ESPAGNE/ WERNER, op. cit. (note 3), pp. 273-326; E. BOTSCH, “Die Bibliothek der Französischen Revolution in Deutschland. Verdeutschungen französischer Revolutionisschriften 1789-1799”, dans: v. ARETIN/ HÄRTER, op. cit. ,pp. 29-36.

36.R. REICHARDT, "Probleme des kulturellen Transfers der Französischen Revolution in der deutschen Publizistik", dans : H. BÖNIG (éd.), Französische Revolution und deutsche Öffentlichkeit. Wandlungen in Presse und Alltagskultur am Ende des 18. Jahrhunderts, München/ London/ New York/ Paris, 1992, p. 138.

37.Sur la problématique des archives interculturelles et la potentialité de ses actes pour une histoire vraiment transnationale, voir les résultats d'un colloque franco-allemand qui a été organisé en 1996 à Leipzig: Michel ESPAGNE/Katharina MIDDELL/Matthias MIDDELL (éds), Archiv und Gedächtnis (Deutsch-Französische Kulturbibliothek, vol. 13), Leipzig, 1999. À partir de ce volume qui sert à la qualification des concepts s'est mis en place un projet commun d'un répertoire des sources interculturelles dans les archives françaises et allemandes dont la responsabilité est partagée entre le Frankreich-Zentrum de l'Université de Leipzig, l'École Normale Supérieure (rue d'Ulm) et l'Institut Historique Allemand à Paris.

38.Hans-Jürgen LÜSEBRINK/Rolf REICHARDT, “Kulturtransfer im Epochenumbruch. Fragestellungen, methodische Konzepte, Forschungsperspektiven”, in: les mêmes (éds), Kulturtransfer im Epochenumbruch Frankreich-Deutschland 1770-1815 (= DeutschFranzösische Kulturbibliothek, vol. 9), Leipzig, 1997, pp. 16-18. Voir aussi leur présentation en français de leur recherche dans "Histoire des concepts et transferts culturels, 1770-1815. Note sur une recherche", Genèses n 14, janvier 1994, pp. 27-42. 39.Il est prévu de publier un CD-ROM qui contient les données du projet sur la sémantique historique selon la comparaison franco-allemande de manière à mettre cet ouvrage énorme d'interprétation des périodiques centrales du temps révolutionnaire aussi à la disposition d'autres investigateurs. Ibid., p. 21, note 27.

40.C. ULBRICH, Transferprozesse in Grenzräumen, dans: Lüsebrink/Reichardt, Kulturttransfer im Epochenumbruch..., pp. 131-137, et K. ANGELIKE/M. BEERMANN/R. NOHR, "Frankophone Zeitungen an der deutschen Westgrenze als Medien des Kulturtransfers”, ibid., pp. 145-192. Une monographie exemplaire sur un des journaux francophones dans la région frontalière: Matthias BEERMANN, Zeitung zwischen Profit und Politik. Der Coruier du Bas-Rhin (1767-1810) (Deutsch-Französische Kulturbibliothek, vol. 4), Leipzig, 1996. 41.M. ESPAGNE, Bordeaux baltique. La présence culturelle allemande à Bordeaux aux XVIIIe et XIXe siècles, Paris, 1991. 
42.F. SCHRADER, “Aufklärungssoziabilität in Bordeaux”, dans: H. E. BÖDEKER/ E. FRANÇOIS (éds), Aufkllärung/Lumières und Politik. Zur politischen Kultur der deutschen und französischen Aufklärung (=Deutsch-Französische Kulturbibliothek, vol. 5), Leipzig, 1996, pp. 249-274.

43.M. ESPAGNE/ M. MIDDELL/ J. GRANDJONC (éds), Transferts culturels et région. L'exemple de la Saxe, (Cahiers d'études germaniques, no 28), 1995.

44.K. MIDDELL, présentation et "Hugenotten in Leipzig - Etappen der Konstruktion einer “hybriden Identität”" et M. ESPAGNE, "Minderheiten und Migration im Kulturtransfer", dans: Th. HÖPEL/ K. MIDDELL (éds), Réfugiés und Emigrés. Migration zwischen Frankreich und Deutschland im 18. Jahrhundert (Comparativ 5-6/1997), Leipzig, 1997, pp. 7-22, 56-75 et 247-258.

45.K. MIDDELL, “Les négociants français à Leipzig et la Révolution française”, in: Sur la Révolution. Approches plurielles (Mélanges Michel Vovelle), Paris, 1997, pp. 423-436. Un résumé provisoire de ses recherches dans: Hugenotten in Leipzig, Leipzig, 1998.

46.Th. HÖPEL, Französische Emigranten in Preußen und Kursachsen, phil. Diss., Leipzig 1999. Pour la France: G. NOIRIEL, "Représentation nationale et catégories sociales. L'exemple des refugiés politiques", Genèses, n² 26, 1997, pp. 25-54.

47.Voir aussi pour la France: S. WAHNICH, L'impossible citoyen. L'étranger dans le discours de la Révolution française, Paris, 1997.

48. La masse des études sur le transfert culturel concerne évidemment le XIX ${ }^{\mathrm{e}}$ siècle, voir par exemple le suivi de ces recherches dans la Revue Germanique Internationale, paraissant depuis 1994 sous la direction de M. ESPAGNE et de J. LE RIDER; voir aussi, dans les Collections philologiques, publ. par M. ESPAGNE/ M. WERNER, vols 1-4, Paris (Éditions de la Maison des Sciences de l'Homme), 1990-1996; la Bibliothèque franco-allemande, dirigée par M. ESPAGNE/ M. WERNER (Éditions du Cerf), Paris, 1991 et suiv. 12 vol. publiés; et la Deutsch-Französische Kulturbibliothek, publiée par Michel ESPAGNE/ Étienne FRANçoIs/ Werner GREILING et Matthias MIDDELL, Leipzig (Leipziger Universitätsverlag), 1993 et suiv., 16 vols parus.

49.Pour le bilan du chantier sur l'histoire régionale en Allemagne voir W. BUCHHOLZ, Landesgeschichte in Deutschland. Bestandsaufnahme - Analysen - Perspektiven, Paderborn, 1998.

50.Cf. par contraste le fort plaidoyer pour une histoire régionale (Landesgeschichte) européenne de W. SCHMALE, Historische Komparatistik und Kulturtransfer. Europageschichtliche Perspektiven für die Landesgeschichte, Bochum, 1998. 51.Voir les exemples dans: M. ESPAGNE/M. MIDDELL, Von der Elbe bis an die Seine..., op. cit. 52.H.-J. LÜSEBRINK/ R. NOHR/ R. REICHARDT, "Die deutsch-französische Übersetzungsbibliothek 1770-1815 ", dans: H.-J. LÜSEBRINK/ R. REICHARDT (éds.), Kulturtransfer im Epochenumbruch. Frankreich - Deutschland 1770 bis 1815, Leipzig, 1997, t. 1, pp. 29-86, surtout pp. 35 et suiv.

53.Cf. les graphiques ibid. pp. 40-43 et 46-48.

54.Ibid., p. 52.

55.Malgré des renseignements multiples sur la diffusion des textes français et leur utilisation dans les sociétés de lecture, etc., malgré de nombreuses suppositions sur la fréquence $d u$ français en tant que langue des cours princières, il reste bien des doutes sur les connaissances réelles du public allemand et sur l'inclination pour des ouvrages en français au lieu des traductions. L'importance des intermédiaires culturels, dont les services de traduction étaient volontiers acceptés et auxquels des recherches récentes 
accordent une attention croissante, montre qu'une certaine connaissance du français ne signifie pas pour autant que la majorité des élites en Allemagne à la fin du XVIII ${ }^{\mathrm{e}}$ siècle se sentirent suffisamment sûres de satisfaire des désirs intellectuels ou politiques dans une langue étrangère. Cf. W. H. STEIN, "Französisch als Sprache der Verwaltungsöffentlichkeit in den rheinischen Departements 1798 bis 1814. Ein aktenkundlicher Befund", dans: LÜSEBRINK/REICHARDT, op. cit., pp. 259-308; W. DAHMEN et al. (éds.), Das Französische in den deutschsprachigen Ländern, Tübingen, 1993 ; J. KRAMER, Das Französische : eine Einführung, Stuttgart, 1992 ; G. SAUDER, "Die französische Sprache in Deutschland in der zweiten Hälfte des 18. Jahrhunderts", dans : Méditionas/ Vermittlungen. Aspekte deutsch-französischer beziehungen vom 17. jahrhundert bis zur Gegenwart, publié par M. GRUNEWALD/ J. SCHLOBACH, Frankfurt a. M., 1992, vol. 2, pp. 97-123. L'évolution conjoncturelle de la parution des journaux francophones en Allemagne peut servir d'indicateur, en effet leur nombre (77 périodiques fondés surtout au XviIIe siècle) reste sensiblement derrière les Pays-Bas (182) et figure de peu avant la Suisse (69); qui plus est il s'agit d'un phénomène surtout attesté dans Vienne (6), Berlin (22) et Francfort sur le Main (17). F. MOREAU, "La presse allemande de langue française 1686-1790. Étude statistique et thématique”, dans: G. SAUDER/ J.SCHLOBACH (éds.), Frankreich und Deutschland im 18. Jahrhundert, Heidelberg, 1986, pp. 244 et suiv; K.ANGELIKE/ M. BEERMANN/R. NOHR, "Frankophone Zeitungen an der deutschen Westgrenze als Medien des Kulturtransfers", dans: Lüsebrink/Reichardt, op. cit., pp. 146-195; M. BEERMANN, Zeitung zwischen Profit und Politik. Der Courir du Bas-Rhin 1767-1810, Leipzig, 1996.

56.LÜSEBRINK/ NOHR/ REICHARDT, article op.cit., p. 59.

57.Voir les graphiques ibid., pp. 76-77.

58.G. ROCHE, “Übersetzen am laufenden Band: zum Beispiel Ludwig Ferdinand Huber \& Co"., dans: Lüsebrink, Reichardt, Kulturtransfer im Epochenumbruch... op. cit, pp. 331-361; sur la famille Huber et les relations de Louis Ferdinand à son père Michael Huber $c f . M$. ESPAGNE, "Übersetzer in Paris und Leipzig: Michael Huber", dans: M. ESPAGNE/ W. GREILING (éds.), Frankreichfreunde. Mittler des deutsch-französischen Kulturtransfers 1750-1850 (Deutsch-Französische Kulturbibliothek, vol. 7), Leipzig, 1996, pp. 85-107. 59.LÜSEBRINK/ NOHR/ REICHARDT, article op.cit., p. 75.

60.M. LEHMSTEDT, “Über den Anteil des Leipziger Buchhandels am literarischen Austausch zwischen Frankreich und Deutschland. Das Beispiel der Peter Philipp Wolfschen Buchhandlung in Leipzig (1795-1803)", dans: LÜSEBRINK/ REICHARD, Kulturtransfer im Epochenumbruch... op. cit. , pp. 403-444; J. FREEDMANN, Zwischen Frankreich und Deutschland. Buchhändler als Kulturvermittler, dans: ibid., pp. 445-498; G. ROCHE, “'Völlig nach Fabrikenart”. Handwerk und Kunst der Übersetzung bei Georg Forster", dans: R. REICHARDT/ G. ROCHE (éds), Weltbürger - Europäer - Deutscher - Franke. Georg Forster zum 200. Todestag, Mainz, 1994, pp. 101-119

61.W. GREILING/M. MIDDELL, "Frankreich-Berichterstattung in Zeitungen: Kursachsen und Thüringen zur Zeit der Französischen Revolution”, dans: LÜSEBRINK/ REICHARDT, Kulturtransfer im Epochenumbruch..., op. cit., pp. 197-238

62.R. NOHR/ E. PAPACEK/ A. VETTER, “'“Das richtige Urtheil über den Zustand der vaterländischen Literatur" ? Zum Anteil des Rezensionswesens an der französischdeutschen Kulturvermittlung im Zeitalter der Aufklärung", ibid., pp. 499-538. 
63.E. PELZER, Die Wiederkehr des girondistischenHelden. Deutsche Intellektuelle als kulturelle Mittler zwischen Deutschland und Frankreich während der Französischen Revolution, Bonn, 1998 (sur la genèse du projet voir pp. 1 et suiv.)

64.Ibid., p. 8.

65.Cf. la bibliographie des traductions étudiées par Pelzer, ibid., pp. 368-480.

66.Ibid., p. 322

67.Ibid., p. 323 : "Introvertiert, den politischen Gedanken und Ideen verpflichtet, waren ihnen Vollblutmenschen, aktionistische politische Macher mit starkem

Entscheidungsimpetus suspekt."

68.R. REICHARDT, Probleme des kulturellen Transfers..., op. cit., p. 91.

69.H.-J. LÜSEBRINK, "Ein Nationalist aus französischer Inspiration: Ernst Moritz Arndt (1769-1860)”, in: M. ESPAGNE/ W. GREILING (éds), Frankreichfreunde..., op. cit. , pp. 221-242. Sur la problématique de la "nationalisation des masses " voir G. L. MOSSE, Die Nationalisierung der Massen. Von den Befreiungskriegen bis zum Dritten Reich, Frankfurt a. M., 1976.

70.Voir p. ex. H.-D. KITTSTEINER, “Was heißt und zu welchem Ende studiert man Kulturgeschichte ?", Geschichte und Gesellschaft 23 (1997), n¹.

71.R. CHARTIER, “Le monde comme représentation”, Annales E.S.C., 1989, nº 6, pp. 1505-1520.

72.D. LANGEWIESCHE, "Nation, Nationalismus, Nationalstaat. Forschungsstand und Forschungsperspektive”, Neue politische Literatur, 1995, pp., 190-236; H. BERDING (éd.), Nationales Bewußtsein und kollektive Identität, Frankfurt a. M., 1994 ; le même (éd.), Mythos und Nation, Frankfurt a. M., 1996, B. GIESEN, Die Intellektuellen und die Nation. Eine deutsche Achsenzeit, Frankfurt a. M., 1993.

73.M. JEISMANN, Das Vaterland der Feinde. Studien zum nationalen Feindbegriff und Selbstverständnis in Deutschland und Frankreich 1792-1918, Stuttgart, 1992.

74.A. DÖRNER, Politischer Mythos und symbolische Politik. Sinnstiftung durch symbolische Formen, Opladen, 1995.

75.JEISMANN, op. cit., pp. 376 et suiv.

76.Е. J. новSBAWM, Nationen und Nationalismus. Mythos und Realität seit 1780, Frankfurt a. M. 1991 (1990); B. ANDERSON, Die Erfindung der Nation. Zur Karriere eines folgenreichen Konzepts, Frankfurt a. M., 1986 (1983) ; M. KOSSOK/ M. MIDDELL, "Mouvements nationaux et enjeux sociaux à l'époque de transition 1500-1850", dans : Nations, Nationalismes, Transitions, $\mathrm{XVI}^{e}-\mathrm{XX}^{e}$ siècle, Paris, 1993, pp. 257-272.

77.Cf. DÖRNER, op. cit., p. 99: “ Die cognitive map des Politischen, die im ancien régime fraglose Gültigkeit besaß, erfährt durch das Erscheinen völlig neuer Akteursgruppen gewaltige Umformungen, der gesamte öffentliche Zeichenraum wird durch und durch politisiert, und es kommt zu einer beschleunigten Verdichtung, Intensivierung und Demokratisierung des gesellschaftlichen Kommunikationsgefüges. "; R. REICHARDT/ B. SCHLIEBEN-LANGE, "Die Französische Revolution als Revolution der Kommunikation und der Sprache", dans: J. GUILHAumou, Sprache und Politik in der Französischen Revolution, Frankfurt a. M., 1989, p. 11.

78.Voir M. WAGNER, “Die Rezeption des “Königsmordes” von 1793 in Deutschland als multimediales Ereignis", dans: LÜSEBRINK/ REICHARDT, Kulturtransfer im Epochenumbruch..., pp. 239-258.

79.K. BAKER/ F. FURET/ C. LUCAS (éds), The French Revolution and the Creation of Modern Political Culture, Oxford et al., 1987-1990, 3 vols. 
80.Bien entendu nous trouvons les traces de cette idée déjà chez Marx, voir par ex. son 18 Brumaire de Louis Bonaparte. Pour une analyse plus détaillée: C. Mossé, L'Antiquité dans la Révolution française, Paris, 1989 après le classique H. T. PARKER, The Cult of Antiquity and the French Revolutionaries, Chicago, 1937.

81.M. vovelLe, L'image de la Révolution française, Paris, 1989, 5 vol.

82.DÖRNER, op. cit., p. 111.

83.Les mêmes conséquences sont analysables dans la guerre de la Vendée et en Espagne.

84.LÜSEBRINK, "Ernst Moritz Arndt...", op. cit. Voir aussi le même dans J. RIESZ (éds), Feindbild und Faszination. Vermittlerfiguren und Wahrnehmungsprozesse in den deutschfranzösischen Kulturbeziehungen 1789-1983, Frankfurt a. M., 1984.

85.D. DÜDING, Organisierter gesellschaftlicher Nationalismus in Deutschland (1800-1847).

Bedeutung und Funktion der Turner- und Sängervereine für die deutsche Nationalbewegung, Tübingen, 1984.

86.DÖRNER, op. cit., p. 124.

87.R. REICHARDT, Das Blut der Freiheit. Französische Revolution und demokratische Kultur, Frankfurt a. M., 1998.

\section{RÉSUMÉS}

L'article décrit le développement de l'historiographie de langue allemande qui, au cours de ces quinze dernières années, est passée d'une comparaison implicite, qui mettait l'accent sur une voie propre à l'Allemagne, à un élargissement du comparatisme classique en histoire sociale à travers la méthode de l'exploitation des transferts culturels. Sont avant tout présentées ici les recherches les plus récentes concernant la réception de la Révolution française dans les fonds allemands de traduction (Deutsche Übersetzungbibliothek), ainsi que l'histoire des médias.

The French Revolution and Germany: from Comparativist Paradigm to the Study of Cultural Transfers. The article describes developments in German-language historiography which over the last fifteen years has undergone a shift from an implicitly comparative approach, typical of the German model, to a broader interpretation of social history using the methodology of cultural transfer research. Recent studies on perceptions of the French Revolution in works translated into German and on the history of the media are highlighted.

L'articolo riassume gli sviluppi dell'istoriografia di lingua tedesca la quale ha avviato una trasformazione del comparatismo classico nel campo della storia sociale (spesso implicito nei suoi termini) viene sottolineata l'esistenza di una via tedesca specifica indirizzata ad un allargamento dei propri strumenti metodologici con un avvicinamento al transfert culturale. Si dà particolare risalto agli studi dedicati alla ricezione della Rivoluzione francese dalla « Deutsche Übersetzungbibliothek » e alla storia dei media.

El artículo resume los desarollos que ocurrieron en la historiografía de los países de habla germana: una transformación del clásico comparatisme de historia social que subrayó la existencia de una vía germana específica hacia una ampliación de sus instrumentos 
metodológicos gracias a los traslados culturales. Se insista ante todo sobre los trabajos centrados sobre la receptión de la revolución francesa y sobre la historia de los media.

Der Artikel beschreibt die Entwicklung der deutschsprachigen Historiographie, die in den letzten 15 Jahren den Übergang von einem impliziten Vergleich, der den Sonderweg Deutschlands betonte, zur Erweiterung des klassischen Komparatismus der Sozialgeschichte im Verfahren der Kulturtransferforschung erlebt hat. Vorgestellt werden vor allem neuere Untersuchungen zur Wahrnehmung der Französischen Revolution in der «Deutschen Übersetzungsbibliothek» und zur Mediengeschichte. 